\title{
Design of Modular FMCW Radar and Quantization of Its Range Error
}

\author{
Mahfoud Y. A. ABDULRAZIGH, Emre UZUNDURUKAN, Ali KARA \\ mahfoud_dc@yahoo.com \\ Atilim University, Electrical and Electronics Engineering \\ uzundurukan.emre@atilim.edu.tr \\ Atilim University, Electrical and Electronics Engineering \\ ali.kara@atilim.edu.tr \\ Atilim University, Electrical and Electronics Engineering
}

Received 02 August 2018; Accepted 03 August 2018; Published online 03 August 2018

\begin{abstract}
This study presents experimental analysis of range error of modular FMCW (frequency modulated continuous wave) radar operating at C-band. As FMCW utilizes frequency modulation, it can measure both range and speed of targets. One of the problems arising in the design of modular FMCW radar is error due to time delays in components and/or modules (Modular Component Delay-MCD). Then, MCD is taken into account to improve the accuracy of range measurement, and range error is quantized. This form of time delay causes frequency offset in measured beat frequency resulting in range error. Therefore, the resulting frequency offset of the radar is estimated and quantized experimentally. In this way, a mean error of about $11 \mathrm{~cm}$ was achieved within $9 \mathrm{~m}$ range measurements for C Band modular FMCW radar.
\end{abstract}

Keywords: modular FMCW radar, C band radar, radar range accuracy, instrumentation radar

\section{Introduction}

Radar is a device to measure range and speed of targets by using radio frequency (RF) signals. As it uses electromagnetic waves, measurements under different conditions such as fog, rain, snow and daytime/night can be achieved. The basic principle is simple; a pulse based transmitting signal (Tx) from the antenna travels through the space and hits objects (targets), and then reflected back to the receiver $(\mathrm{Rx})$ antenna. Finally, both signals are processed to give the range to the objects and their speeds if not stationary [1].

Naturally, CW (Continues Wave) radar can only measure the speed but not the distance. Therefore, improvement is needed to measure both range and speed. Then, the bandwidth of $\mathrm{CW}$ signal can be increased by utilizing frequency and/or phase modulation (for example, FMCW). The modulation signal might be a triangle, sawtooth, sinusoidal, or more complex waveform.

As discussed in [2], Phase Loop Lock (PLL) has been widely used in FMCW radar systems. In this way, linear frequency modulated (LFM) signal with $2 \mathrm{GHz}$ bandwidth can be generated easily. PLL can provide a great linearity over a broad bandwidth. Consequently, it can achieve high precision of range measurement. On the other hand, prototype hardware can easily be developed by implementing signal processing functions on Field Programmable Gate Array (FPGA) and/or Digital Signal Processor (DSP). A radar system has been reported in [2]. In [3], a concept to increase the accuracy of range measurement is presented. It is based on the elimination of the internal noise and compensation of large path loss. For this purpose, amplification of the signal at beat frequency through a Gain Variable Amplifier (GVA) is proposed.

In [4], a software based FPGA implementation is reported. It affects the precision of range measurement due to the VCO's linearity. The software defined radio interface increases frequency by several $\mathrm{kHz}$ in every step of the counter according to the distance. It provides higher linearity for the modulated signal within the tuning voltage range. Moreover, instead of FFT the arc cosine memory unit has been used in signal processing block. In this way, the range is calculated in the time domain, and the computational 
complexity of the hardware is reduced. An interesting approach has been proposed in [5]. It combines two modulation types, namely LFM and FSK. Then it uses the benefit of them in order to achieve higher unambiguous range and high-resolution velocity measurement. Also, it reduces the computation time. Moreover, it enhances the precision even in multi-target situations. Hence it improves the performance of the radar.

This study presents design considerations of a modular FMCW radar for use in laboratory and experimental researches. More specifically, it quantifies the range error along with the range resolution by examining time delays due to modular components. The paper is organised as follows; the next section summarizes the operation of of FMCW radar. Section 3 describes design considerations for FMCW radar where emphasis is given to modular components delays (MCD). Section 4 presents radar implementation followed by the range measurement tests in the laboratory. Finally, conclusions are drawn.

\section{Operation of FMCW Radar}

In the literature, two simple block diagrams are widely used to illustrate the principles of FMCW radar; single antenna and double antenna FMCW radars. Figure 1 shows double antenna FMCW block diagram. It utilizes two antennas for receiving and transmitting signals. This provides many advantages.

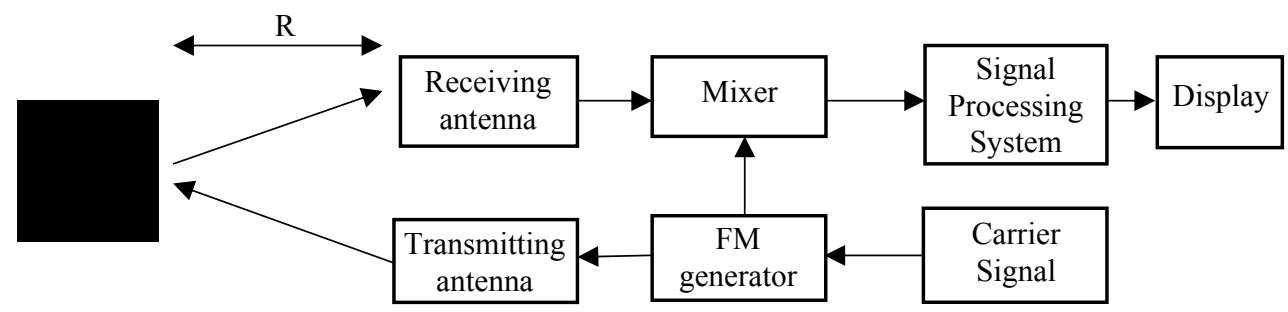

Figure 1 Simplified block diagram of bistatic FMCW radar

Distance between receiving and transmitting antennas is critical as it decreases possible leakage from the transmitting channel to the receiving channel to an insignificant level. Typically, very small amount of the transmitted signal will reflect back as an echo signal. Then, a weak sample of the transmitted signal should go into the mixer. The mixer generates the intermediate frequency (IF) signal. IF signal may typically haave two centers; the sum and diffrence of the inputs, e.i., Tx and Rx channels. In order to eliminate high frequencies components (the sum), typically, a low pass filter (LPF) is used. Then, the difference signal component can be obtained to extract the signal components from which the range and speed information can be derived.

\subsection{FMCW Radar Signaling}

FMCW radar emits continuously similar to a simple $\mathrm{CW}$ radar. However, it varies the frequency over a period of time regularly. Thus, it is improved version of typicall $\mathrm{CW}$ waveform as it uses modulating signal. The modulation signal might be sawtooth, triangular or sinusoidal. In general, such modulated radar waveform is called chirp signal. It allows to measure both the speed and the range of the target, simultaneously. Use of chirp signal in FMCW radar can be illustrated as in Figure 2 where frequency variation of Tx signal is taken linear.

Considering Figure 1 and 2, let us try to formulate the mechanism in range and/or speed measurement. Once the FMCW signal is transmitted, an echo signal reflecting from a target at the range $\mathrm{R}$ will appear at the input of the receiving antenna. The propagation delay between the echo/reflected and the transmitted signals can be calculated by

$\tau=\left(\frac{2 R}{c}\right)$

where $\boldsymbol{c}$ is the speed of light, $\boldsymbol{\tau}$ is the propagation delay and $\boldsymbol{R}$ is the range from the radar antenna to the target. 


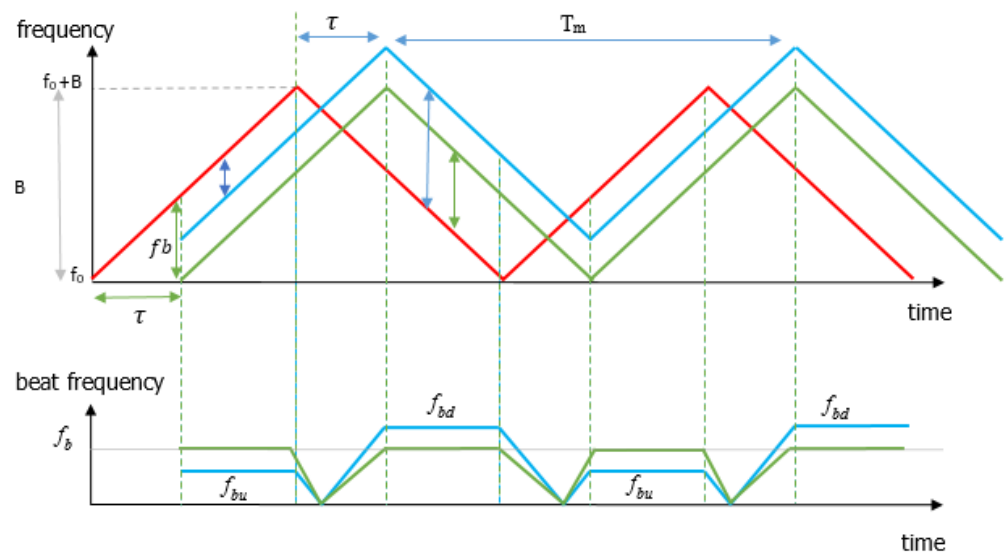

Figure 2 Transmitted, two received signals (modulation frequency) and two beat frequencies according to received signals.

If the target is stationary, the level of the frequency of the difference signal at the output of the mixer (the beat frequency) only correlates to the range and is given by

$\frac{f_{b}}{\tau}=\frac{B}{T_{m} / 2}$

where $\boldsymbol{B}$ is the modulation bandwidth and $\boldsymbol{T}_{\boldsymbol{m}}$ is the period of triangular signal (modulation period). On the other hand, for a moving target, the Doppler effect drifts the absolute values of the received frequencies. As a result, the following can be written

$f_{d u}=f_{b}-f_{d}$

$f_{b d}=f_{b}+f_{d}$

where $\boldsymbol{f}_{\boldsymbol{d}}$ is the Doppler frequency, $\boldsymbol{f}_{\boldsymbol{d} \boldsymbol{u}}$ and $\boldsymbol{f}_{\boldsymbol{b} \boldsymbol{d}}$ are the beat frequencies of rising and decaying slopes of the modulation waveform. Once the levels of the beat frequency is measured in the baseband, the range and the relative speed of the target can be obtained from

$R=\frac{c\left(f_{b u}+f_{b d}\right) T_{m}}{8 B}$

$V r=\frac{c\left(f_{b d}-f_{b u}\right)}{4 f_{c}}$

where $\boldsymbol{f}_{\boldsymbol{c}}$ is the center frequency of transmitted signal over the rising and decaying slopesas fwmulated in [6].

The minimum distance that can be measured between two targets is referred to range resolution. As the minimum time duration $\boldsymbol{\tau}_{\text {min }}$, between Tx and Rx signals, is

$\tau_{\min }=\frac{1}{B}$

The range resolution, $\Delta \boldsymbol{R}$, for the radar is then

$\Delta R=\frac{c}{2 B}$

From which the beat frequency can be calculated as

$f_{b}=\frac{4 B R}{c T_{m}}$.

Therefore, the beat frequency is directly proportional to the range. Therefore, the range can be obtained according to the beat frequency [7]. 


\subsection{FMCW Signal Simulations (AWR/VSS)}

The best way in design of RF front end is to use high level system blocks. A design tool called virtual system simulator (VSS) provided by AWR is very attractive to do this. It is used to analyse radar waveforms and system level signal flows in radar front end. A typical block diagram in simulating FMCW signal is shown in Figure 3. Figure 4 shows the power spectrum of a typical FMCW signal. Both are generated in AW/VSS environment.

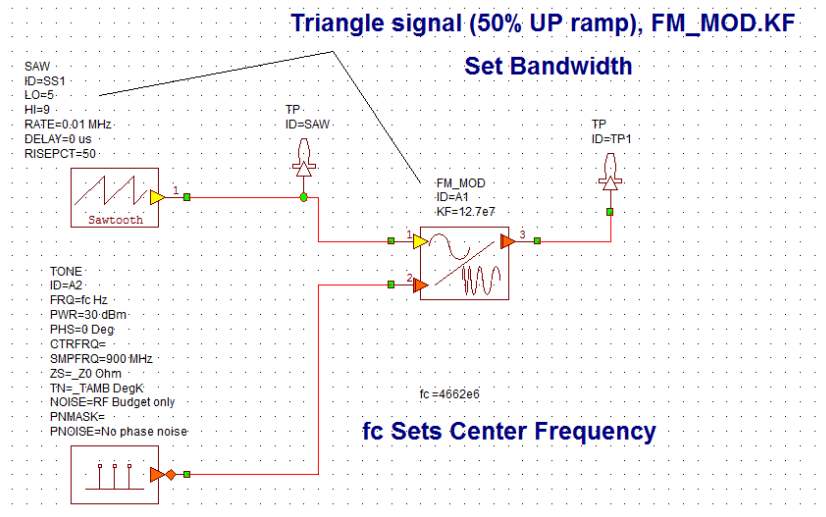

Figure 3 Block diagram of FMCW signal Tx model in AWR/VSS

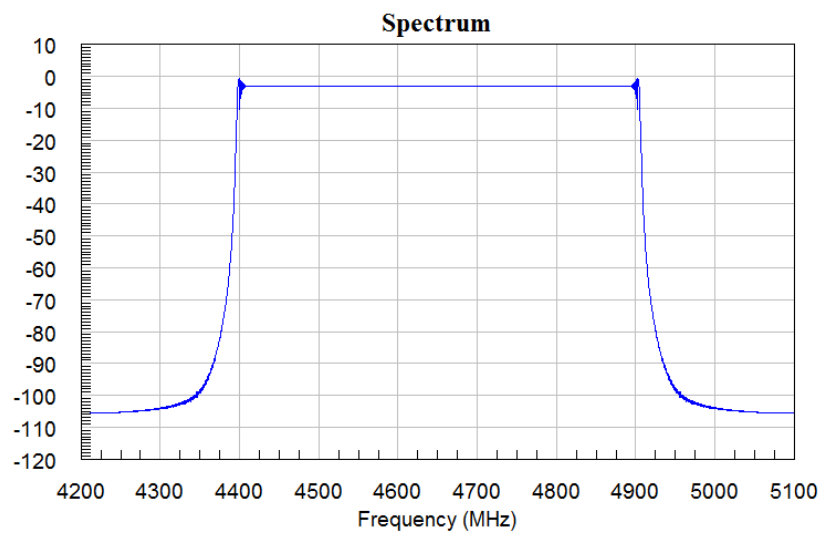

Figure 4 The power spectrum of the modulated signal (FMCW)

From the hardware point of view, the simplest way to generate an FMCW signal is to use a VCO along with arbitrary waveform generator (AWG). Using modular VCO components, it is practical to generate FMCW signals in the laboratory. Figure 5 shows the measured spectrum of such FMCW signal by the spectrum analyzer in the lab environment. In reference with the radar operational blocm diagram, this signal represents the power spectrum of the signal at the output of the VCO (FM generator). The challenge in generating a linear FMCW signal is the linearity of VCO over the operational bandwidth. 


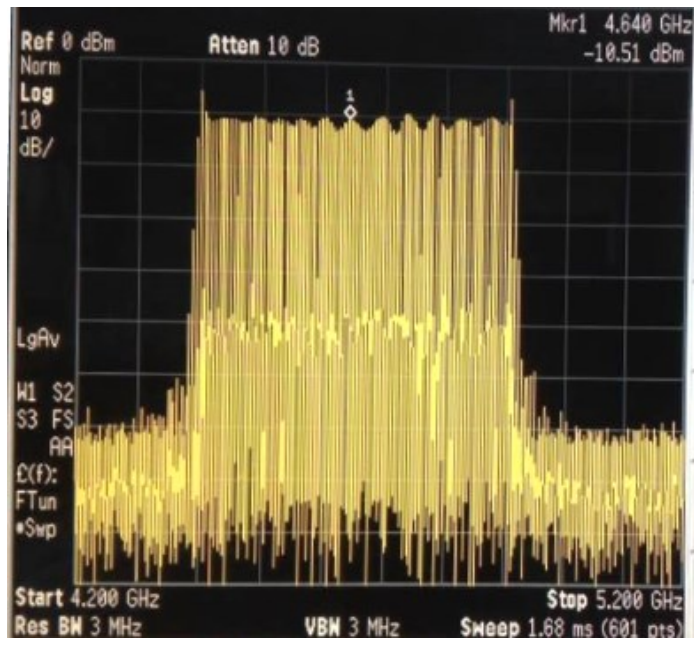

Figure 5 The measurement of FMCW spectrum

\section{FMCW Radar Design}

Operational block diagram of FMCW radar was described previously. This section considers some of the design parameters of a FMCW radar. The requirements are taken simple; greater indoor range, higher range resolution and accuracy of the range measurement. First of all, two antenna configuration was chosen in order to provide higher isolation between the channels and so enhance the sensitivity of the receiver.

\subsection{Radar Range}

Radar range is dependent many system level parameters. The objective of this paper is not to describe each instead explain how each is chosen appropriately. By adopting range equation derived by a stepby step basis, here the firnal form of range equation will be provided $[1,8,9]$. The maximum range is proportional to the minimum detectable signal (MDS) from

$R_{\text {max }}=\left(\frac{P_{t} G_{t} G_{r} \lambda^{2} \sigma}{(4 \pi)^{3} M D S L_{s y s}}\right)^{1 / 4}$

where $\boldsymbol{R}_{\text {max }}$ is the maximum range, $\boldsymbol{P}_{\boldsymbol{t}}$ is the transmit power, $\boldsymbol{\sigma}$ is the radar cross section (RCS) of the target, $\boldsymbol{\lambda}$ is the operational wavelength, $\boldsymbol{L}_{\boldsymbol{s} y \boldsymbol{s}}$ is overall losses in the system, $\boldsymbol{G}_{\boldsymbol{t}}$ is the transmit antenna gain and $\boldsymbol{G}_{\boldsymbol{r}}$ is the receive antenna gain. This is called instrumentation range of the radar. For a targeted RCS, all aother parameters can be tuned for maximum range.

\subsection{The Minimum Detectable Signal (MDS)}

The capability of radar receivers in detecting weak signals is limited by the power of the Rx noise along with the power of the echo signal. The weakest signal that can be detected is called MDS (Minimum Detectable-or discernable- Signal). It is a key parameter for a radar system to quantify the maximum distance, and also it defines the radar receiver sensitivity. MDS is highly dependent on receiver noise as formulated by

$M D S=10 \log _{10}\left(\frac{k T_{0}}{1 m W}\right)+N F_{\text {sys }}+10 \log _{10} B W+S N R_{\text {out }}$

where $\boldsymbol{k}$ is well-known Boltzmann's constant, $\boldsymbol{T}_{\mathbf{0}}$ is the reference noise temperature $\left(290^{\circ} \mathrm{K}\right), \boldsymbol{N F}_{\boldsymbol{s y s}}$ is the noise figure of the receiving system, $\boldsymbol{B} \boldsymbol{W}$ is the receiver bandwidth $(\mathrm{Hz}), \boldsymbol{S} \boldsymbol{N} \boldsymbol{R}_{\text {out }}$ is the minimum 
required SNR for detection. A typical signal-to-noise ratio (SNR) depends on the probability of false alarm (Pfa) rate and the probability of detection (Pd) of the radar system. Typical values of these parameters are $10^{-6}$ and $95 \%$, respecitively, as in many radar literature book, as in [1,9].

\subsection{Noise Figure (NF)}

Noise figure or noise factor (NF or F) is a parameter describing the level of the noise generated by a system. Some common noise types could be thermal noise, shot noise, and other noise effects due to the physics of different types of circuit components. All of these noise effects are combined to give noise factor (F). Apparently, NF degrades the SNR (Signal to Noise Ratio) for a given input signal power. Apparently, NF is precisely provided for the components like LNA and IF amplifiers by the manufacturer or the designer. Each device in a signal chain contributes to the overall receiver noise figure of the system according to the following

$N F_{s y s}=10 \log _{10}\left(F_{1}+\frac{F_{2}-1}{G_{1}}+\frac{F_{3}-1}{G_{1} G_{2}}+\cdots+\frac{F_{n}-1}{G_{1} G_{2} \ldots G_{n}}\right)$

where $\boldsymbol{F}_{\boldsymbol{n}}$ is the noise factor and $\boldsymbol{G}_{\boldsymbol{n}}$ is the gain of each stage in the component chain of the system.

A portion of a typical receiver is shown in Figure 6. To estimate the cascaded noise figure of this receiver part, all NF's and the gains of each blokc have to be taken into account in the last equation.

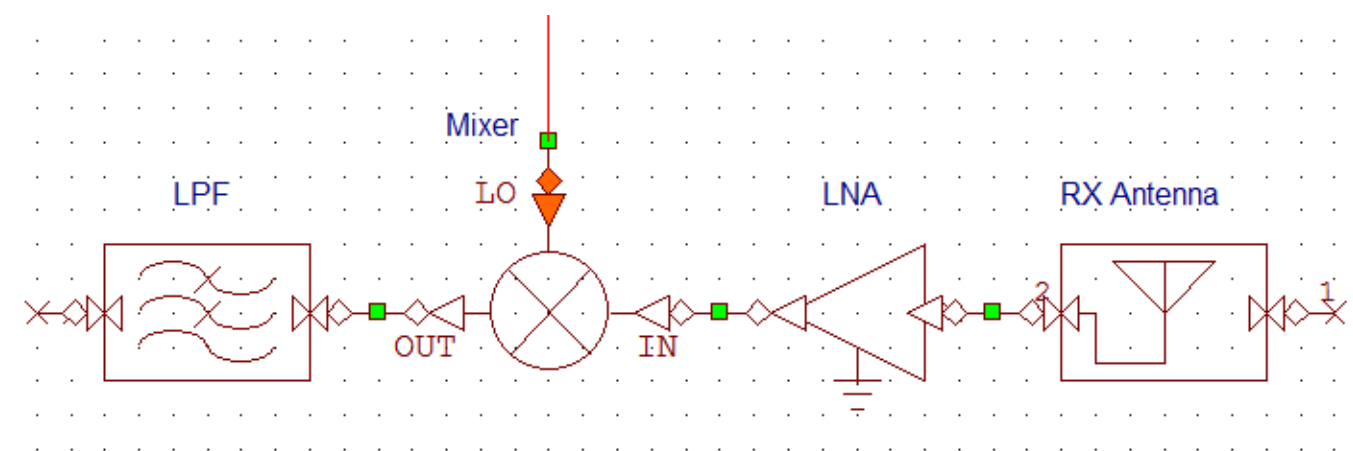

Figure 6 Receiver Part

\subsection{Velocity Factor}

It is a measure how fast the signal can travel through the line. As known, RF signal travels in cables slower than free space. Therefore, the ratio of actual velocity of propagation through the cable and the speed of light in vacuum refer to velocity factor. There is a direct relationship between velocity of propagation $(\boldsymbol{v})$ and the operational wavelength $(\lambda)$

$v=f \lambda$

At high frequencies, the velocity factor is given by

$v_{p}=\frac{v}{c}=\frac{1}{\sqrt{\epsilon_{e f f}}}$

where the velocity factor is a dimensionless parameter $\boldsymbol{v}_{\boldsymbol{p}}$ (less than unity), and $\boldsymbol{v}$ is the velocity of propagation in the medium (m/s), $\boldsymbol{c}$ is the speed of light in free space and $\boldsymbol{\epsilon}_{\boldsymbol{e f f}}$ is the effective dielectric constant of the medium. Then, dielectric properties of cables and their lengths are critical for the time delays in the system. This time delay causes a shift in the frequency. Any shift in the frequency is resulted in range error or ambiguity. Then, it is worth noting how this velocity factor affects range error. 


\subsection{Modular Component Delay (MCD)}

All circuits or systems suffer from some form of the time delay between the input and the output. If the signal is applied to the circuit, then there is time elapsed to get the output. That time is called time delay or time constant in general. As known, the modular RF components such as PA, LNA, splitter and mixer are compact electronic modules or circuits. Hence, they may include passive and active components such as capacitor, inductor, resistor, diode and transistor. Thus, if the delays due to these elements are unknown, measurements in the system will be ambiguous. Alternatively, this ambiguity can be resolved by precise calibrations.

Now, consider VSS model of a modular radar front end design shown in Figure 7. It can be seen that the signals are transmitted from Tx to Rx antenna via reflecting from an object (target) at a distance R. Therefore, the time delay occurs in space during the traveling of the signals. Moreover, as explained previously, an offset time delay should be expected due to the cables, PA, LNA and Tx, Rx antenna. These time delays are considered in the measurement of beat frequency as it introduces an offset to the the actual beat frequency, as given by

$f_{m u}=f_{b}+f_{\text {offset }}$

where the measured frequency is $\boldsymbol{f}_{\boldsymbol{m} \boldsymbol{u}}$, and $\boldsymbol{f}_{\text {offset }}$ is the offset frequency. Apparently, this offset frequency causes errors in the range measurement.

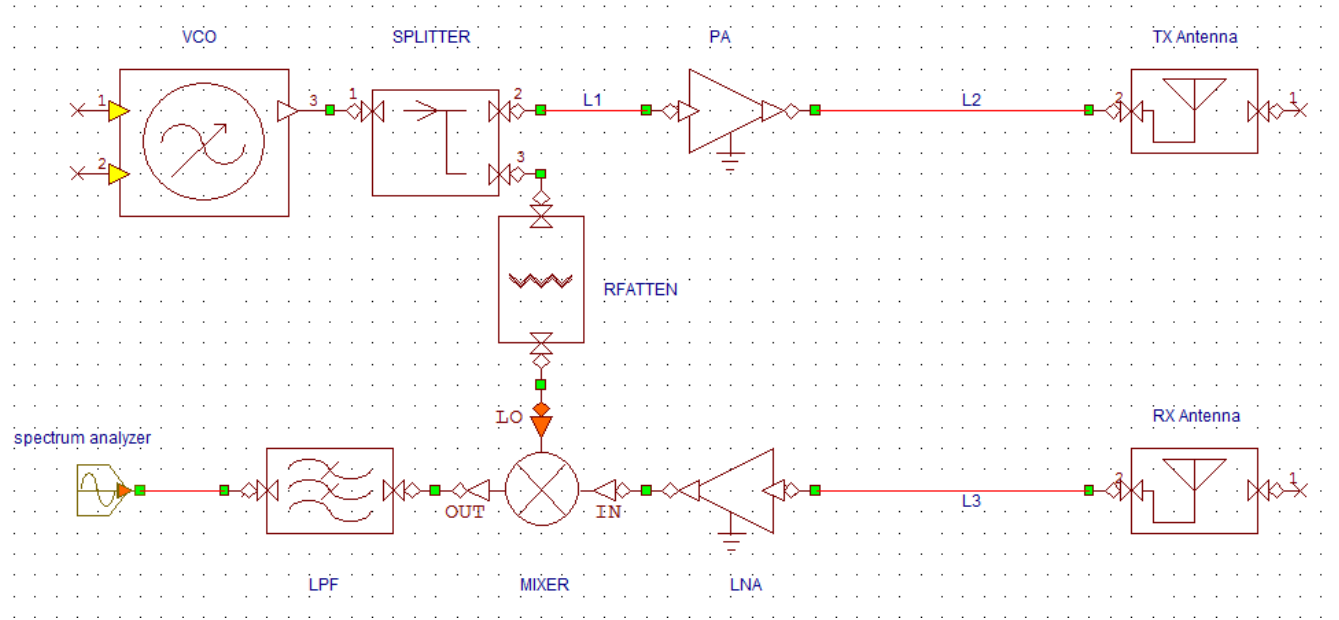

Figure 7 FMCW Radar Simulation Model (AWR/VSS)

Considering the time delays in each component of the system in Figure 7, the modular component delay or instrumentation delay can be split up into

$\tau_{I D}=\tau_{L 1}+\tau_{P A}+\tau_{L 2}+\tau_{T x}+\tau_{R x}+\tau_{L 3}+\tau_{L N A}$

where the total time delay of the instruments is $\tau_{I D}$ and $\tau_{L 1}, \tau_{L 2}, \tau_{L 3}$ are the time delays of the cables separately. $\boldsymbol{\tau}_{T x}$ is Tx antenna time delay, $\boldsymbol{\tau}_{R x}$ is Rx antenna time delay, $\boldsymbol{\tau}_{P A}$ is the time delay of the power amplifier (PA) and $\boldsymbol{\tau}_{L N A}$ is the LNA time delay. From the Equation 3, overall offset frequency can be obtained as

$f_{\text {offset }}=\frac{2 \tau_{I D} B}{T_{m}}$

Note that the time delay due to coaxial cables should be determined by taking into acoount the velocity factor provided in the datasheet, or by using time domain reflectometer (TDR) measurement. Thus, as the length of cables is known, the cable offset frequency can be obtained from

$f_{c o}=\frac{2 B L}{v_{p} T_{m}}$

This could easily be simulated in AWR/VSS with reaslitic figures. No more details will be given here. 


\section{Radar Implementation}

Figure 1 and Figure 7 shows the block diagram and AWR/VSS model of the radar implemented in this work. In radar implementation, one of the challenge was to obtain linearity in FMCW signal. It is not easy to achieve better resolution without greater bandwidth. To achieve the highest bandwith, it is necessary to use higly linear VCO, or PLL. To minimize the cost, there is on option; use of highly linear VCO. The best possible VCO has been chosen from the manufacturer product catalog. Figure 9 shows the linearity of the VCO chosen for this radar design. The highest bandwidth we can achieve was about $500 \mathrm{MHz}$ over $4400 \mathrm{MHz}$ to $4900 \mathrm{MHz}$. The output frequency of the VCO, we choose, can vary "linearly" in this range according to input tuning voltage.

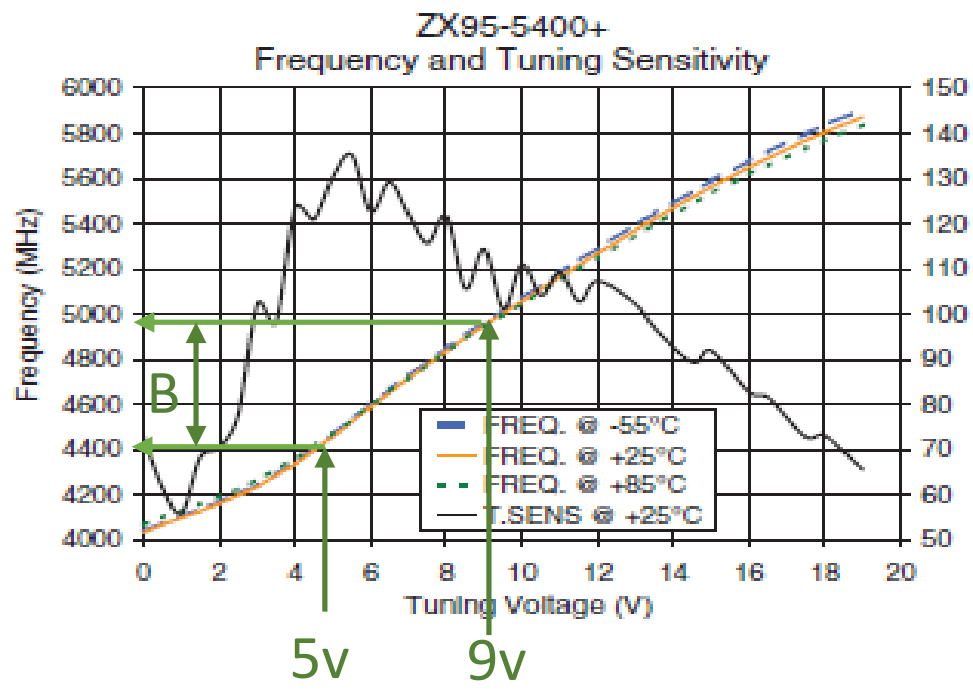

Figure 9 VCO output frequency versus tuning voltage [10]

There were two solutions for supplying waveform generator to the VCO. One was to use digitally synthesized signals using simple PIC. The other was to use laboratory instruments, that is, arbitrary waveform geneator (AWG). As will be shown later, for the targeted radar specs, the modulating triangular signal has $100 \mu \mathrm{s}$ sweep time (period). After that, the output of the VCO then is divided by a splitter. One output of the splitter is connected to the mixer after a $10 \mathrm{~dB}$ attenuator as a reference signal for the received signal chain. The other output is connected to the power amplifier (PA) to feed the transmitting antenna. The details of the design is described in the first named author's thesis work.

\section{Range Measurements}

A simple test has been conducted to estimate the performance of FMCW radar in indoor laboratory environment. The specifications of the implemented FMCW radar is shown in Table 1.

Table 1 FMCW radar specifications

\begin{tabular}{|c|c|}
\hline Frequency & $4.4 \sim 4.9 \mathrm{GHz}$ \\
\hline Bandwidth & $525 \mathrm{MHz}$ in C-Band \\
\hline Range Resolution & $\sim 0.29 \mathrm{~m}$ \\
\hline Sweep type & Triangular \\
\hline Sweep time & $100 \mu \mathrm{s}$ \\
\hline Target Range & $1.2 \sim 9 \mathrm{~m}(0.6 \mathrm{~m}$ step) \\
\hline Target & C-Band Horn Antenna \\
\hline Antennas & RG 58 \\
\hline Cables (coax cable) & \\
\hline
\end{tabular}


Two identical horn antennas are used in transmit and receive chains. Each antenna has $15 \mathrm{dBi}$ gain at 4.6GHz. The operation of the FMCW radar system was validated with a $1 \mathrm{~m}^{2}$ flat aluminum plate as a target. The target is placed in front of the antennas with $60 \mathrm{~cm}$ steps from $1.2 \mathrm{~m}$ to $9 \mathrm{~m}$. The beat frequency is measured according to the distance of the target by taking MCD into account at every step. If we take a closer look at the parameteric dependencies; according to Equation 12 noise figure of receiver cascaded has been calculated as $2.1 \mathrm{~dB}$, MDS is specified to be $-75 \mathrm{dBm}$ according to Equation 11, and then the maximum range is about 16 meters with a target of $1 \mathrm{~m}^{2} \mathrm{RCS}$ according to Equation 10.

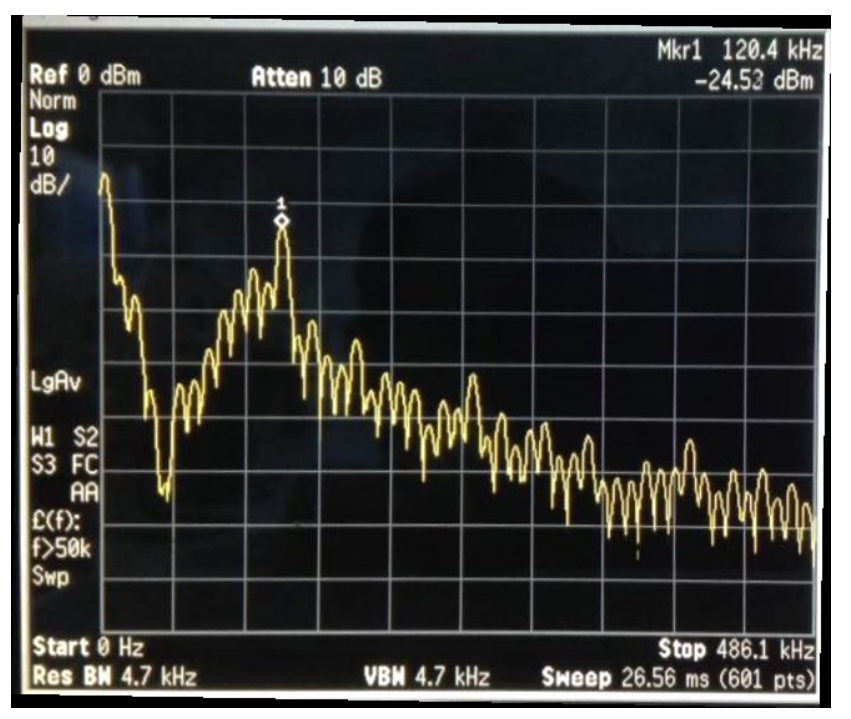

Figure 10 Measurement Offset frequency

Measurement of the offset frequency was the next stage, and it was $120 \mathrm{kHz}$ as illustrated in Figure 10. However, offset introduced by the cables is $102 \mathrm{kHz}$ by Equation 18 where $\boldsymbol{v}_{\boldsymbol{p}}=66 \%$ is taken, and the overall length of the cables was $1.93 \mathrm{~m}$. This means that the offset frequency due to the PA \& LNA combination was about $18 \mathrm{kHz}$. Since the time delays in the antennas are not included in the measured values, the overall offset frequency should be slightly higher than $120 \mathrm{kHz}$.

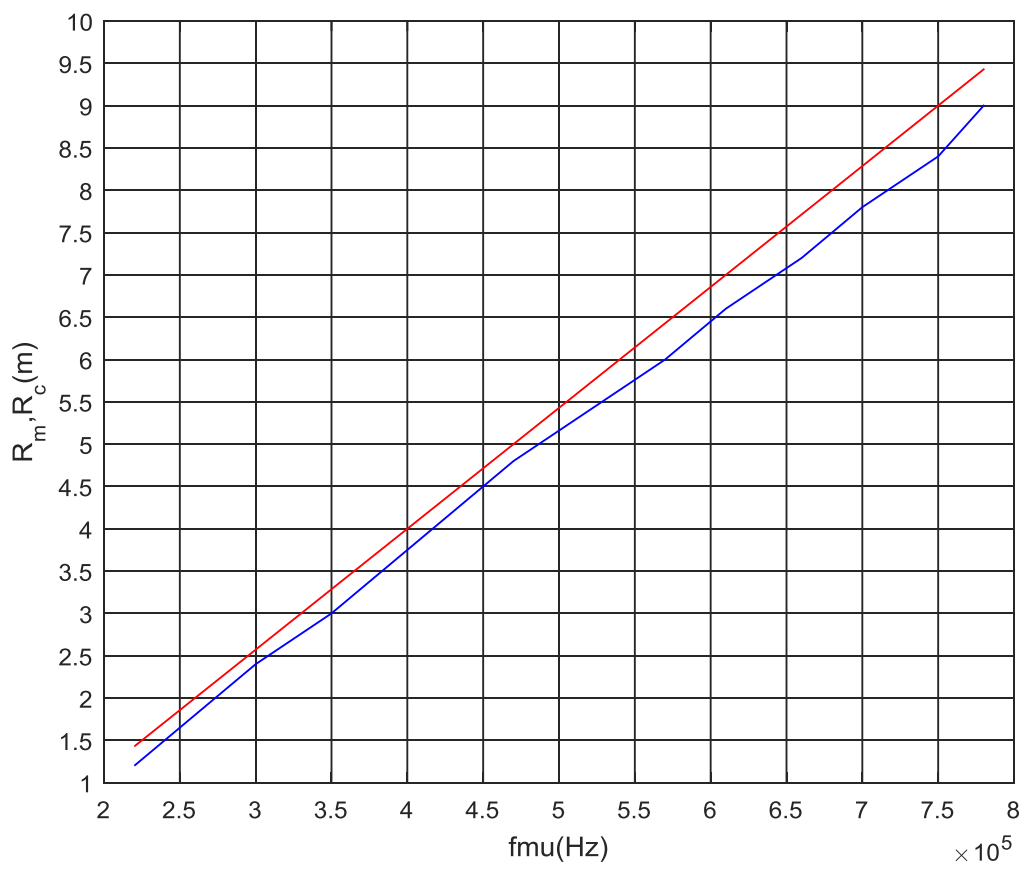

Figure 11 Measured range with measured beat frequency by removing the overall frequency offset 
By removing frequency offsets introduced by time delays, the accuracy of the radar has been higly improved. Figure 11 shows the beat frequency versus the range after eliminating the overall offset frequencys. A shown, the average error was about $10 \mathrm{~cm}$. Note that in the figure, the blue line denotes the measurement results while the red line denotes the expected results.

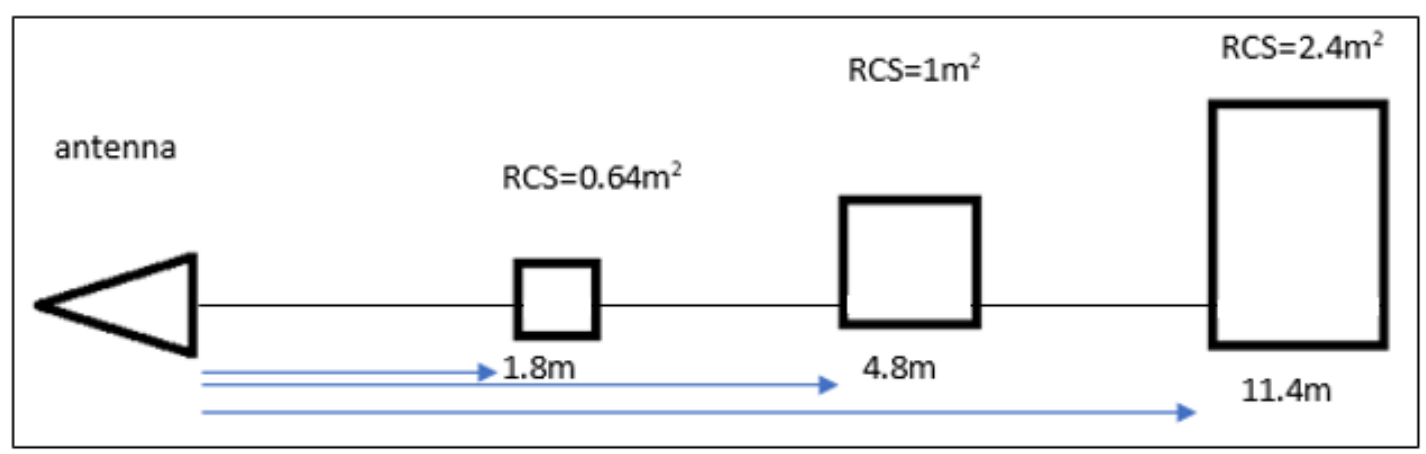

Figure 12 Range resolution tests (three targets with different RCSs and ranges)

The last test was the rnge resolution of thr radar. For this purpose, three targets are placed at the different disrances over a line, a shown in Figure 12. The RCS and the range of the targets are balanced to have comparable signal powers due to the instrumental range of the radar.

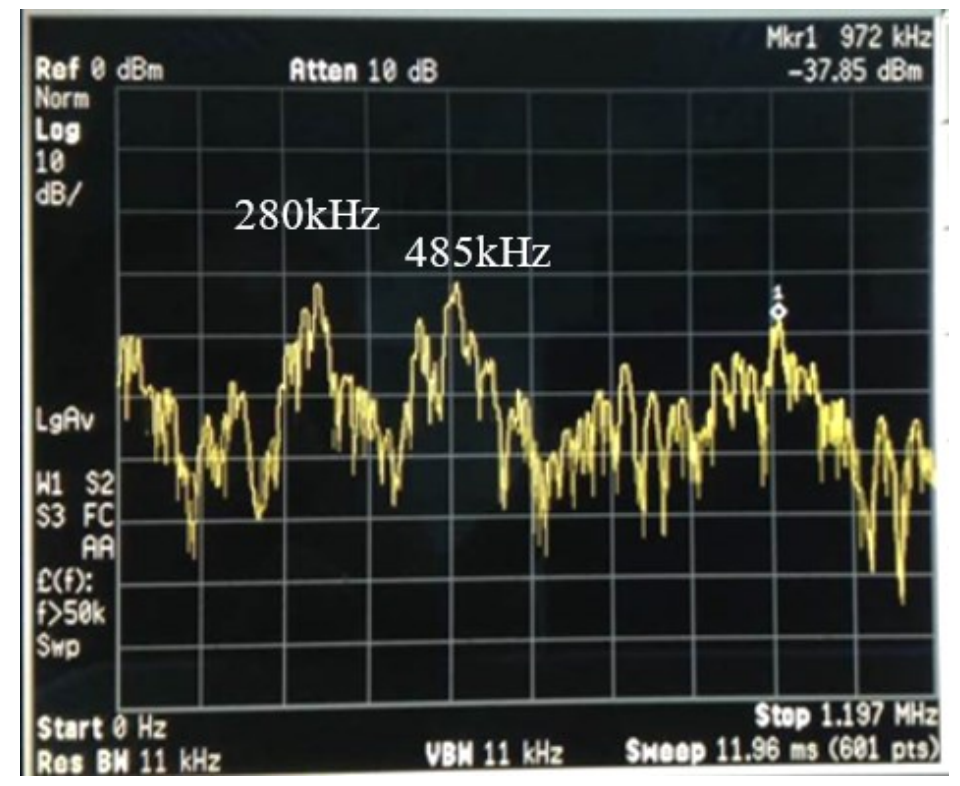

Figure 13 Beat frequency for the three targets

Figure 13 shown the screenshot of the spectrum analyzer where three distinctive beat frequencies belonging to the targets are clearly visible. Although this should still be studied, this prelimnary tests demonstrates that the radar front end works well

\section{Conclusion}

In this study, design and implementation of a modular FMCW radar operating at $\mathrm{C}$ band are discussed. Moreover, the range accuracy and resolution of the radar are improved. The range accuracy of such a modular radar is highly dependent on delays between and in-component delays. The range resolution is improved by increasing the bandwidth of the radar signals. Some preliminary measurements were conducted in the laboratory to estimate the range accuracy and the range resolution of the FMCW radar. 
The range accuracy of the FMCW radar systems depends on the beat frequency measured at the output of the mixer. The beat frequency is also proportional to the traveling time from the antenna to the target along with the time delays imposed by modular components. This time delay can be called MCD (Modular Component Delay). The MCD of the FMCW radar designed in this work was studied quantitatively in the laboratory. Then the mean value and the standard deviation of the range error was less than $10 \mathrm{~cm}$ within $9 \mathrm{~m}$ indoor range. The bandwidth of FMCW radar was also extended to $525 \mathrm{MHz}$ in order to achieve a range resolution of less than $30 \mathrm{~cm}$.

For portable operation of the FMCW, it is possible to implement a FPGA board instead of instrumentation (the spectrum analyzer). This could be future work for improvements of the radar system.

\section{References}

[1] M. I. Skolnik, "Introduction to radar systems," McGraw-Hili book company, 1962.

[2] E. Hyun, Y. S. Jin, and J. Lee, "Development of $24 \mathrm{GHz}$ FMCW level measurement radar system," in Radar Conference, 2014 IEEE, 2014.

[3] D. H. Shin and S. O. Park, "Design of X-band FMCW short range radar," in Intelligent Radio for Future Personal Terminals (IMWS-IRFPT), 2011 IEEE MTT-S International Microwave Workshop Series on, 2011, IEEE.

[4] H. Lim and S. Lee, "A Short Range FMCW Radar System with Low Computational Complexity," Int. J. Electron. Electrical Eng, 4(4), 2016, pp. 370-373.

[5] H. Rohling and M. M. Meinecke, "Waveform design principles for automotive radar systems," in Radar, 2001 CIE International Conference on, Proceedings, 2001, IEEE.

[6] B. Dagdeviren, K. Y. Kapusuz and A. Kara, "A modular FMCW radar RF front end design: Simulation and implementation," in Signal Processing and Communications Applications Conference (SIU), 2014 22nd, 2014, IEEE.

[7] B. R. Mahafza, "Radar systems analysis and design using MATLAB" 2002, CRC press.

[8] M. Y. A. Abdulrazigh, E. Uzundurukan and A. Kara, "Analysis of Measurement Instrumentation Delay in Modular Experimental Radar at C Band," in Signal Processing and Communications Applications Conference (SIU), 2018 26th, 2018, IEEE.

[9] G. L. Charvat, "Small and short-range radar systems," 2014, CRC Press.

[10] Mini-Circuits. Datasheet. 2016; Available from: https://ww2.minicircuits.com/pdfs/ZX95$5400+$.pdf. 